\title{
Activation of soluble guanylate cyclase by nitric oxide in
} lymphocytes correlates with minimal hepatic encephalopathy in cirrhotic patients

\author{
Vicente Felipo ${ }^{2}$, Blanca Piedrafita ${ }^{2}$, Amparo Urios², Miguel A Serra ${ }^{1}$, A del \\ Olmo $^{1}$, José M Rodrigo ${ }^{1}$ and Carmina Montoliu*1
} \author{
${ }^{2}$ Laboratory of Neurobiology, Centro de Investigacion Principe Felipe, Valencia, Spain \\ Email: Carmina Montoliu* - carmina@cipf.es \\ * Corresponding author

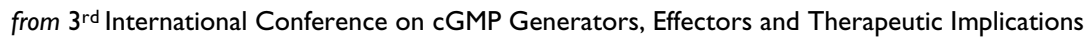 \\ Dresden, Germany. I5-17 June 2007 \\ Published: 25 July 2007 \\ BMC Pharmacology 2007, 7(Suppl I):PI8 doi:I0.II86/I47I-22I0-7-SI-PI8
}

Address: ${ }^{1}$ Servicio de Hepatología, Hospital Clínico Universitario, Departamento de Medicina, Universidad de Valencia, Valencia, Spain and

This abstract is available from: http://www.biomedcentral.com/I47/-22/0/7/SI/PI8

(C) 2007 Felipo et al; licensee BioMed Central Ltd.

\section{Background}

Altered modulation of cGMP levels in brain seems to be responsible for the impairment of some types of cognitive function [1]. The homeostasis of cGMP is also strongly altered in blood in patients with liver disease, who show increased plasma cGMP but reduced cGMP content in lymphocytes [2]. Activation of soluble guanylate cyclase by NO is also altered in lymphocytes of patients with liver cirrhosis [2]. This suggests that alterations in cGMP homeostasis in blood could reflect the alterations in brain and be therefore associated with the appearance of minimal hepatic encephalopathy (MHE). The aim was to assess whether there is a correlation between the alterations in different parameters involved in modulation of cGMP levels and the presence of MHE.

\section{Methods}

We studied in 46 patients with liver cirrhosis and 26 controls the performance in the PHES battery of psychometric tests, critical flicker frequency (CFF), cGMP in plasma and lymphocytes, activation of guanylate cyclase by NO in lymphocytes, ammonia, nitric oxide metabolites and atrial natriuretic peptide (ANP).

\section{Results}

Activation of guanylate cyclase by NO in lymphocytes and cGMP in plasma were higher and CFF lower in patients with than without MHE. Ammonia, ANP and metabolites of nitric oxide were higher in patients than in controls but were no different in patients with or without MHE (Figures 1, 2, 3, 4; Table 1).

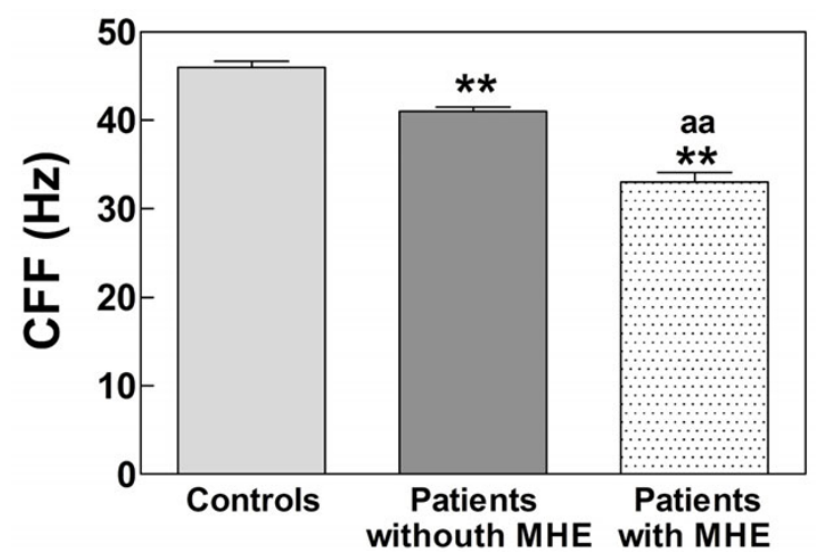

Figure I

Critical flicker frequency. 


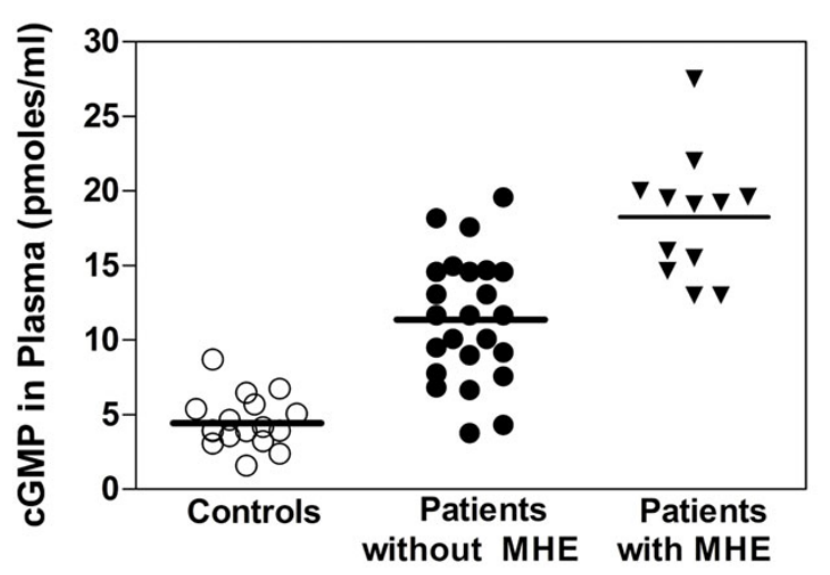

Figure 2

Plasma cGMP.

\section{Conclusion}

Alteration in activation of guanylate cyclase by NO in lymphocytes correlates with PHES performance, CFF and ammonia levels (Table 2). This suggests that altered modulation of guanylate cyclase by NO in lymphocytes would reflect a parallel alteration in brain occurring in patients with MHE that would be involved in their cognitive impairment.

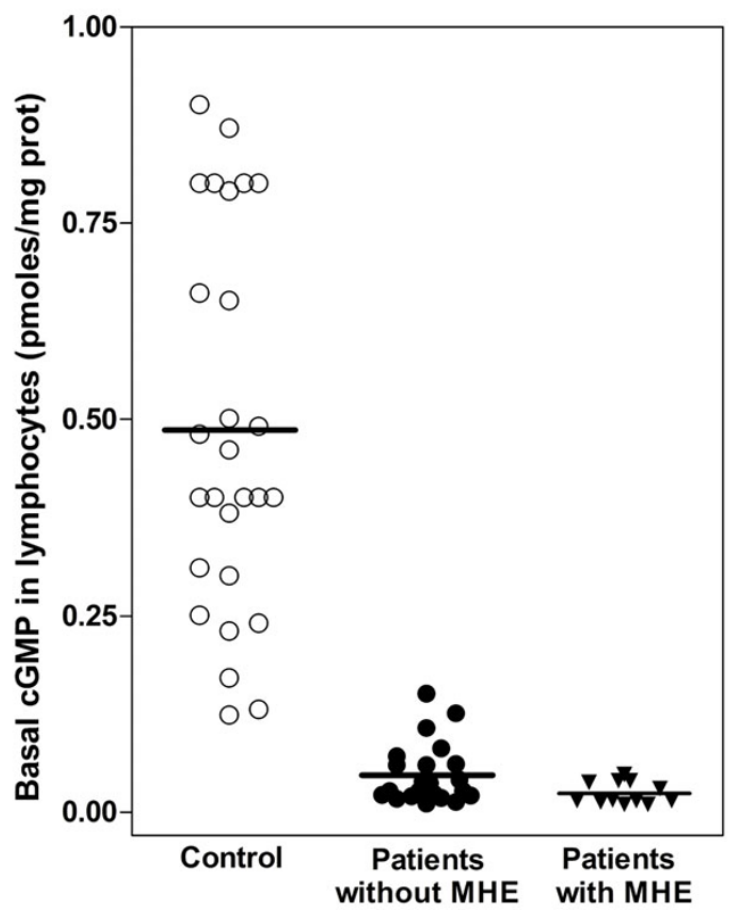

Figure 3

Basal cGMP in lymphocytes.

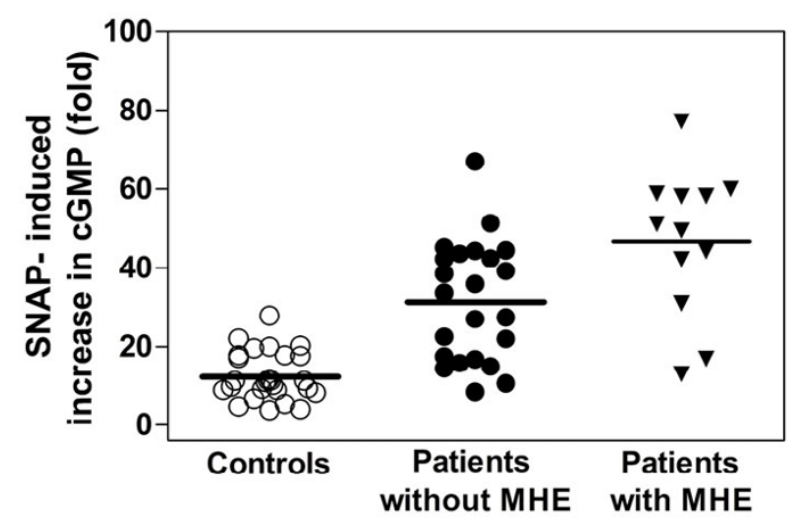

Figure 4

NO-induced increase in cGMP in lymphocytes. 
Table I: Values of the different parameters analyzed in controls and in patients.

\begin{tabular}{|c|c|c|c|c|}
\hline PARAMETERS & CONTROLS Media \pm SD (n) & $\begin{array}{c}\text { PATIENTS WITHOUT MHE p } \\
\text { vs. Control }\end{array}$ & $\begin{array}{c}\text { PATIENTS WITH MHE P } \\
\text { vs. control }\end{array}$ & $\begin{array}{l}\text { PATIENTS WITH MHE p } \\
\text { vs. without MHE }\end{array}$ \\
\hline $\begin{array}{l}\text { Basal cGMP in lymphocytes } \\
\text { (pmoles/mg prot) }\end{array}$ & $\begin{array}{l}0.47 \pm 0.15 \\
(n=26)\end{array}$ & $\begin{array}{c}0.049 \pm 0.02 \\
(\mathrm{n}=22) \mathrm{P}<0.001 * *\end{array}$ & $\begin{array}{c}0.025 \pm 0.015 \\
(\mathrm{n}=12) \mathrm{P}<0.001 * *\end{array}$ & $p>0.05$ \\
\hline $\begin{array}{l}\text { SNAP-induced cGMP increase } \\
\text { (fold) }\end{array}$ & $\begin{array}{l}12 \pm 6 \\
(n=26)\end{array}$ & $\begin{array}{c}31 \pm 15 \\
(n=23) p<0.001 * *\end{array}$ & $\begin{array}{c}47 \pm 18 \\
(n=12) p<0.001 * * * *\end{array}$ & $\mathrm{P}<0.05 *$ \\
\hline cGMP in Plasma $(\mu \mathrm{M})$ & $\begin{array}{l}4.4 \pm 1.7 \\
(n=17)\end{array}$ & $\begin{array}{c}12 \pm 4 \\
(\mathrm{n}=24) \mathrm{p}<0.001 * *\end{array}$ & $\begin{array}{c}18 \pm 4 \\
(\mathrm{n}=12) \mathrm{p}<0.00 \mid * * *\end{array}$ & $\mathrm{P}<0.001 * *$ \\
\hline Nitrates + Nitrites $(\mu M)$ & $\begin{array}{l}22 \pm 9 \\
(n=26)\end{array}$ & $\begin{array}{c}31 \pm 13 \\
(n=31) \mathrm{p}<0.05^{*}\end{array}$ & $\begin{array}{c}38 \pm 14 \\
(n=13) p<0.001 * * * 2\end{array}$ & $\begin{array}{c}P=0.09 \\
(n s)\end{array}$ \\
\hline ANP in Plasma $(\mathrm{pg} / \mathrm{ml})$ & $\begin{array}{l}78 \pm 13 \\
(n=13)\end{array}$ & $\begin{array}{c}119 \pm 25 \\
(\mathrm{n}=14) \mathrm{P}<0.001 * *\end{array}$ & $\begin{array}{c}140 \pm 50 \\
(\mathrm{n}=9) \mathrm{P}<0.00 \mid * *\end{array}$ & $\begin{array}{l}P=0.193 \\
(n s)\end{array}$ \\
\hline Ammonia concentration $(\mu \mathrm{M})$ & $\begin{array}{l}76 \pm 30 \\
(n=23)\end{array}$ & $\begin{array}{c}156 \pm 86 \\
(n=23) p=0.001 * * *\end{array}$ & $\begin{array}{c}|5| \pm 51 \\
(n=9) p<0.00 \mid * * *\end{array}$ & $\begin{array}{c}P=0.89 \\
(n s)\end{array}$ \\
\hline CFF $(\mathrm{Hz})$ & $\begin{array}{l}46 \pm 3 \\
(n=26)\end{array}$ & $\begin{array}{c}41 \pm 3 \\
(\mathrm{n}=24) \mathrm{P}\end{array}$ & $\begin{array}{c}33 \pm 4 \\
(n=15) p<0.001 * *\end{array}$ & $\mathrm{p}<0.001 * *$ \\
\hline SCORE PHES & $\begin{array}{l}-0.15 \pm 1 \\
(n=26)\end{array}$ & $\begin{array}{c}-0.57 \pm 1 \\
(\mathrm{n}=31)\end{array}$ & $\begin{array}{c}-8.7 \pm 2.1 \\
(n=15)\end{array}$ & $\mathrm{P}<0.001 * *$ \\
\hline Age & $43 \pm 12$ & $50 \pm 8$ & $55 \pm 11$ & $\begin{array}{l}P>0.05 \\
(n s)\end{array}$ \\
\hline
\end{tabular}

Table 2: Correlations between different parameters analysed.

\begin{tabular}{|c|c|c|c|}
\hline \multicolumn{4}{|c|}{ CORRELATION WITH } \\
\hline PARAMETER & PHES & CFF & AMMONIA \\
\hline cGMP in plasma & $\begin{array}{l}\text { NO } \\
P=0.06, r=-0.235\end{array}$ & $\begin{array}{l}\text { YES } \\
P=0.004, r=-0.368\end{array}$ & $\begin{array}{l}\text { YES } \\
P=0.011, r=0.386\end{array}$ \\
\hline cGMP in lymphocytes & $\begin{array}{l}\text { NO } \\
p=0.67, r=-611\end{array}$ & $\begin{array}{l}\text { YES } \\
p=0.029, r=-0.281\end{array}$ & $\begin{array}{l}\text { NO } \\
p=0.28, r=-0.165\end{array}$ \\
\hline $\begin{array}{l}\text { Activation of sGC by } \\
\text { NO }\end{array}$ & $\begin{array}{l}\text { YES } \\
P=0.004, r=-0.369\end{array}$ & $\begin{array}{l}\text { YES } \\
P<0.0001, r=-0.544\end{array}$ & $\begin{array}{l}\text { YES } \\
P=0.003, r=0.433\end{array}$ \\
\hline Ammonia & $\begin{array}{l}\text { NO } \\
P=0.28, r=-0.156\end{array}$ & $\begin{array}{l}\text { YES } \\
P=0.021, r=0.333\end{array}$ & \\
\hline
\end{tabular}

\section{References}

I. Erceg S, Monfort P, Hernandez-Viadel M, Llansola M, Montoliu C, Felipo $\mathrm{V}$ : Restoration of learning ability in hyperammonemic rats by increasing extracellular cGMP in brain. Brain Res 2005, 1036: II5-121.

2. Montoliu C, Kosenko E, Del Olmo JA, Serra MA, Rodrigo JM, Felipo $V$ : Correlation of nitric oxide and atrial natriuretic peptide changes with altered cGMP homeostasis in liver cirrhosis. Liver Int 2005, 25:787-795.

Publish with Bio Med Central and every scientist can read your work free of charge

"BioMed Central will be the most significant development for disseminating the results of biomedical research in our lifetime. "

Sir Paul Nurse, Cancer Research UK

Your research papers will be:

- available free of charge to the entire biomedical community

- peer reviewed and published immediately upon acceptance

- cited in PubMed and archived on PubMed Central

- yours - you keep the copyright

Submit your manuscript here:

http://www.biomedcentral.com/info/publishing_adv.asp
BioMedcentral 